\title{
Streambed microstructure predicts evolution of development and life history mode in the plethodontid salamander Eurycea tynerensis Ronald M Bonett ${ }^{* 1,2}$ and Paul T Chippindale ${ }^{1}$
}

Address: ${ }^{1}$ Department of Biology, University of Texas at Arlington, Arlington TX 76019, USA and ${ }^{2}$ Museum of Vertebrate Zoology and Department of Integrative Biology, University of California, Berkeley, CA 94720, USA

Email: Ronald M Bonett* - rbonett@berkeley.edu; Paul T Chippindale - paulc@uta.edu

* Corresponding author

Published: 02 March 2006

BMC Biology2006, 4:6 doi:10.1186/174I-7007-4-6

This article is available from: http://www.biomedcentral.com/I741-7007/4/6

(c) 2006Bonett and Chippindale; licensee BioMed Central Ltd.

This is an Open Access article distributed under the terms of the Creative Commons Attribution License (http://creativecommons.org/licenses/by/2.0), which permits unrestricted use, distribution, and reproduction in any medium, provided the original work is properly cited.

\begin{abstract}
Background: Habitat variation strongly influences the evolution of developmentally flexible traits, and may drive speciation and diversification. The plethodontid salamander Eurycea tynerensis is endemic to the geologically diverse Ozark Plateau of south-central North America, and comprises both strictly aquatic paedomorphic populations (achieving reproductive maturity while remaining in the larval form) and more terrestrial metamorphic populations. The switch between developmental modes has occurred many times, but populations typically exhibit a single life history mode. This unique system offers an opportunity to study the specific ecological circumstances under which alternate developmental and life history modes evolve. We use phylogenetic independent contrasts to test for relationships between a key microhabitat feature (streambed sediment) and this major life history polymorphism.
\end{abstract}

Results: We find streambed microstructure (sediment particle size, type and degree of sorting) to be highly correlated with life-history mode. Eurycea tynerensis is paedomorphic in streams containing large chert gravel, but metamorphoses in nearby streams containing poorly sorted, clastic material such as sandstone or siltstone.

Conclusion: Deposits of large chert gravel create loosely associated streambeds, which provide access to subsurface water during dry summer months. Conversely, streambeds composed of more densely packed sandstone and siltstone sediments leave no subterranean refuge when surface water dries, presumably necessitating metamorphosis and use of terrestrial habitats. This represents a clear example of the relationship between microhabitat structure and evolution of a major developmental and life history trait, and has broad implications for the role of localized ecological conditions on larger-scale evolutionary processes.

\section{Background}

The ability to express alternate phenotypes enables populations to colonize and persist in variable environments [1-4]. However, the role of phenotypic plasticity in the evolution of traits, and subsequent diversification and speciation, is understood in relatively few instances [4].
Populations experiencing a given environmental regime across generations may undergo directional selection, from a highly plastic trait to a single condition [4-6], thus initiating phenotypic divergence among populations that occupy different environments. Furthermore, selection acting on reproductive traits may lead to reproductive iso- 
lation among populations. Especially for organisms with complex life cycles, local selection involving major developmental shifts can produce rapid changes in ecology, morphology and life history among populations, and may hasten divergence and speciation [7-9].

Amphibians exhibit diverse life history strategies that enable them to exploit both aquatic and terrestrial ecosystems successfully [10-13]. Most amphibians have a free-living aquatic larval stage followed by metamorphosis into a more terrestrial adult form [10-13]. One deviation from this pathway, exhibited by some salamanders, is paedomorphosis, whereby individuals reach adulthood while maintaining many larval structures (particularly gills) and an aquatic lifestyle $[10,14]$. In some groups of salamanders the switch between paedomorphosis and metamorphosis can be highly labile, and a multitude of ecological and evolutionary trade-offs accompany each strategy [extensively reviewed in [15-17]]. The most important advantage of metamorphosis is the ability to leave ephemeral aquatic habitats $[11,15,18,19]$, and in some situations metamorphosis could potentially increase gene flow between otherwise isolated aquatic systems [20]. In permanently aquatic environments, especially those of high quality, paedomorphosis may be optimal [11,15,21-24]. Also, paedomorphs remain in their breeding habitat, limiting mortality from breeding migrations and providing the chronological advantage of early arrival $[23,25]$. In regions where peripheral habitats are extremely harsh or dry, remaining in the aquatic environment may be the only viable strategy $[11,26-28]$.

The Ozark Plateau of south-central North America is a geologically diverse highland with many endemic species [29-31]. Watercourses carve through the plateau at several distinct stratigraphic levels, causing the deposition of different types of sediment among streams [32]. Silurian/ Ordovician chert strata are very hard and deposit as loosely associated gravel in many western Ozark streams, whereas in most other localities stream sediment is composed of densely packed clastic sediments such as sandstone and siltstone. The interstitial spaces within chert gravel beds harbour a diverse fauna, including a variety of arthropods, small fishes, and salamanders. Streambed microstructure is critical for small aquatic organisms in these communities, because it provides pathways to underground water during harsh dry summer conditions, when the surface can dry completely [29,33-35].

The plethodontid salamander Eurycea tynerensis is restricted to the Ozark Plateau, and, until very recently, was considered to be a strictly paedomorphic species, with a distribution closely associated with Silurian/Ordovician chert gravel streams in the western Ozarks [33-36]. However, recent molecular phylogenetic evidence revealed that

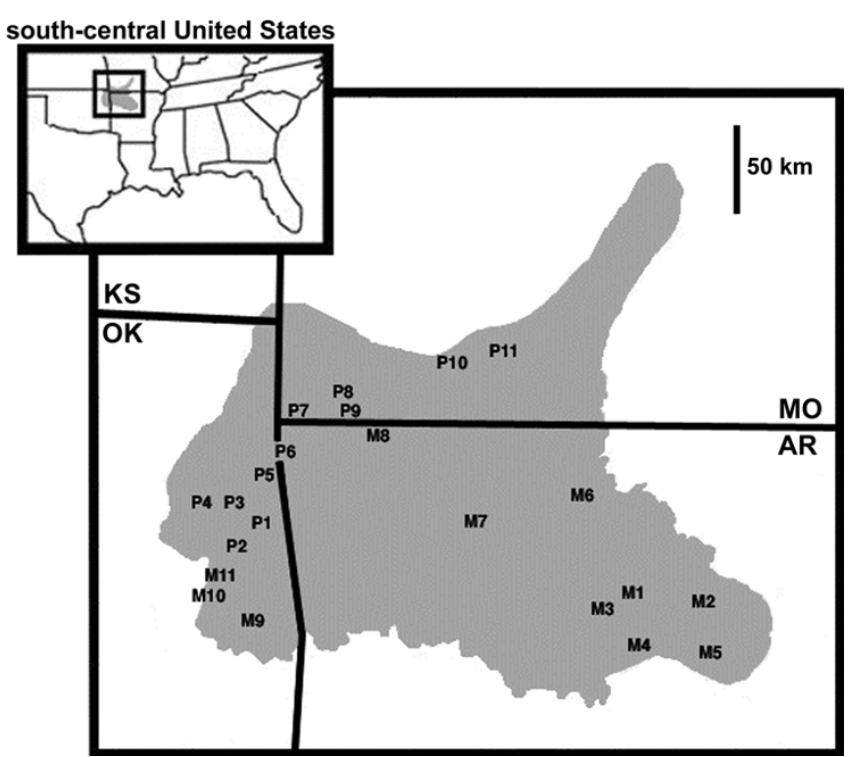

Figure I

Distribution of $E$. tynerensis and localities sampled. Geographic distribution of Eurycea tynerensis (shaded) in the south-central United States. Locality codes correspond to those in Additional File I, and indicate life history mode ( $M=$ metamorphic and $\mathrm{P}=$ paedomorphic). The states Arkansas, Kansas, Missouri and Oklahoma are abbreviated AR, KS, MO and OK, respectively.

E. tynerensis is composed of paedomorphic and metamorphic populations (the latter formerly considered $E$. multiplicata griseogaster), and paedomorphosis appears to have arisen independently many times [37]. Populations of $E$. tynerensis characteristically exhibit a single life-history mode, suggesting that local habitat parameters are highly influential, via either selection for a facultative response or for particular genotypes [37]. This system offers a unique opportunity to investigate the role of local microhabitat conditions responsible for major phenotypic divergence.

Here we investigate the importance of streambed microstructure in the evolution of a major life-history polymorphism. We quantify substrate size, degree of sorting and type for 22 populations (11 paedomorphic and 11 metamorphic; Fig. 1) of E. tynerensis from across the Ozark Plateau. Using a phylogeny based on 1,818 bp of the mitochondrial genes $c o b$ and nad4 to assess relationships among populations, we test for correlations between substrate parameters (size, sorting and type) and life-history mode (paedomorphic vs. metamorphic) using phylogenetic independent contrasts. On the basis of our results we make inferences about the evolution and maintenance of alternate life-history modes in this group across the geologically diverse Ozark Plateau. Furthermore, we discuss the role of microhabitat in initiating local divergence, 


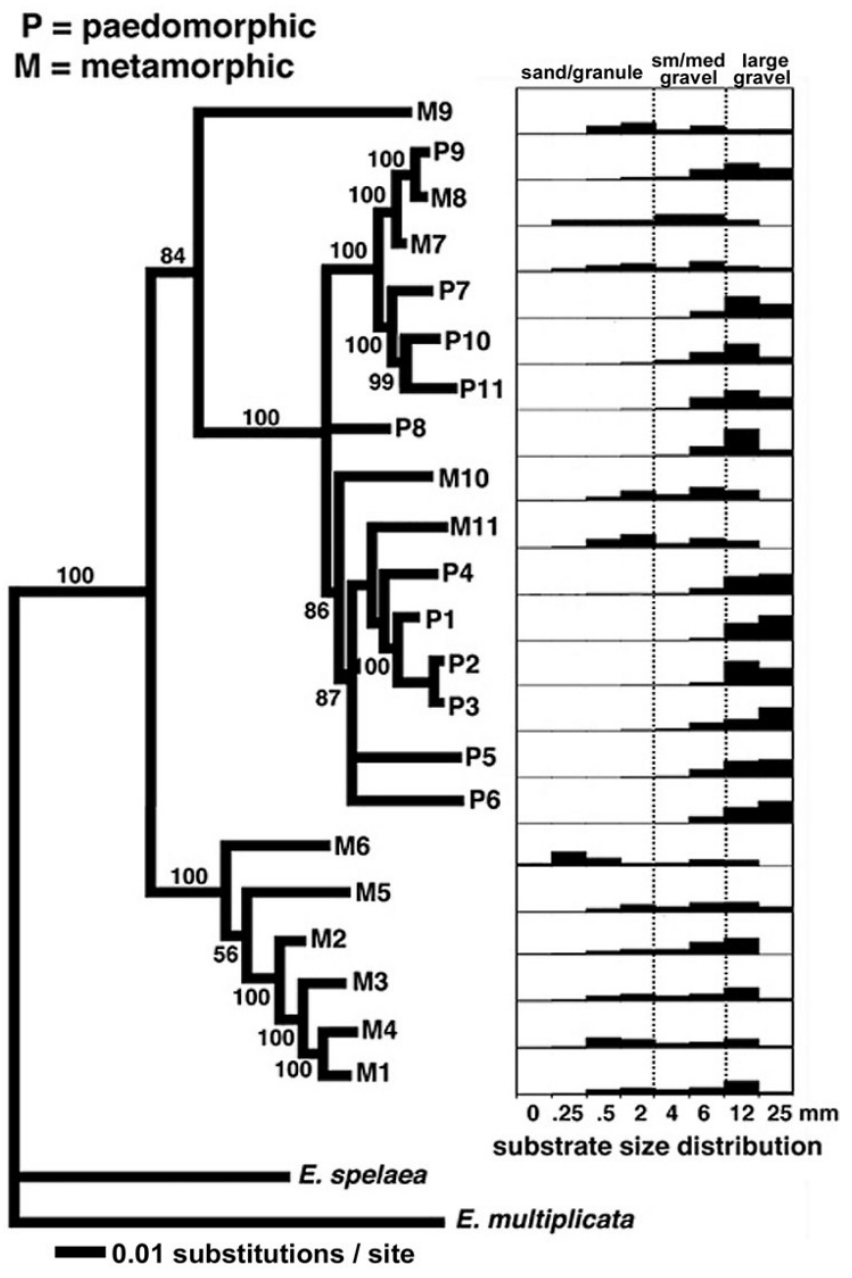

Figure 2

Phylogeny and size distribution of substrate samples. Bayesian phylogeny of 22 populations of $E$. tynerensis. Topology is based on a majority rule consensus of 50,000 trees ( 5 $\times 10^{6}$ generations sampled every 100 generations). Branch lengths were averaged across 50,000 trees. Posterior probabilities indicate node support, and only those $\geq 50 \%$ are shown. Histograms show proportions of substrate in each size class for each locality. Boundaries of the three size categories: sand and granule ( 0 to $<2 \mathrm{~mm}$ ), small/medium gravel $(>2$ to $<12 \mathrm{~mm})$ and large gravel $(\geq 12 \mathrm{~mm})$ are indicated with dotted lines.

which ultimately may result in large-scale changes in patterns of organismal development, morphology, ecology and life history, as well as formation of new species.

\section{Results}

\section{Sequence variation and phylogeny}

The concatenated $c o b$ and nad4 sequences for all individuals formed an alignment of 1,818 homologous nucleotides. Each individual displayed a unique haplotype and uncorrected pairwise sequence divergence ranged from 0.22 to $9.35 \%$ among the 22 populations of E. tynerensis. Bayesian analysis yielded a well-resolved tree with strong support for most relationships (Fig. 2). Maximum parsimony analysis and nonparametric bootstrapping (not shown) yielded very similar, well-supported trees. Phylogenetic independent contrasts calculated using the topology of the Bayesian tree were nearly identical to those based on the maximum parsimony topology. Given the similarity between the results of both methods, and since Bayesian analyses provide a well-parameterized, modelbased estimation of branch lengths, we only show the results based on the Bayesian tree.

\section{Independent contrasts of substrate parameters}

The distribution of substrate size differed in metamorphic (M) versus paedomorphic (P) localities (Fig. 2). Most notably, P localities lack substrate of the smaller size classes. Phylogenetic independent contrasts (PICs) [3840 ] are powerful methods that account for ancestry when testing for relationships between putative adaptations, or adaptations and the environment. For each locality we determined the proportion of sediment in three broad size categories: sand/granule ( 0 to $<2 \mathrm{~mm})$, small/ medium gravel $(2$ to $<12 \mathrm{~mm})$, and large gravel $(\geq 12$ $\mathrm{mm}$ ). Using PICs we found a significant negative correlation between paedomorphosis and the presence of material in the sand and granule $\left(\mathrm{r}^{2}=0.7056 ; p<0.001\right.$; Fig. $3 \mathrm{~A})$ and small/medium gravel $\left(\mathrm{r}^{2}=0.3721 ; p<0.002\right.$; Fig. 3B) categories.

Sorting is a measure of the uniformity of particle sizes in a substrate sample [41], and is an important parameter for determining overall streambed compactness (embeddedness $[33,34])$. Streambeds composed of well sorted large material provide open spaces between particles, whereas weakly sorted streambeds (containing multiple size classes) are much less penetrable because small material fills in any interstitial spaces (see also discussion of embeddedness below). To determine the degree of sorting, we counted the number of size classes ( 1 to 8 ) represented in the substrate samples at each locality. The size class had to represent at least $5 \%$ of the total substrate sample in order to be counted. The substrate in P localities is very well sorted (i.e. composed of few size classes), whereas $M$ localities had substrate in many size classes. We found a strong negative correlation between the number of size classes and the presence of paedomorphs $\left(\mathrm{r}^{2}=0.7396 ; p<0.001 ;\right.$ Fig. 3C), indicating that substrate particles were well sorted in $\mathrm{P}$ and poorly sorted in $\mathrm{M}$ localities. Our samples were primarily composed of clastic rocks (sandstone and siltstone) and chert. Clastic rocks are composed of smaller particles (e.g., sand and silt), while chert is an extremely hard siliceous substance. Eurycea tynerensis previously was found to be closely associated 

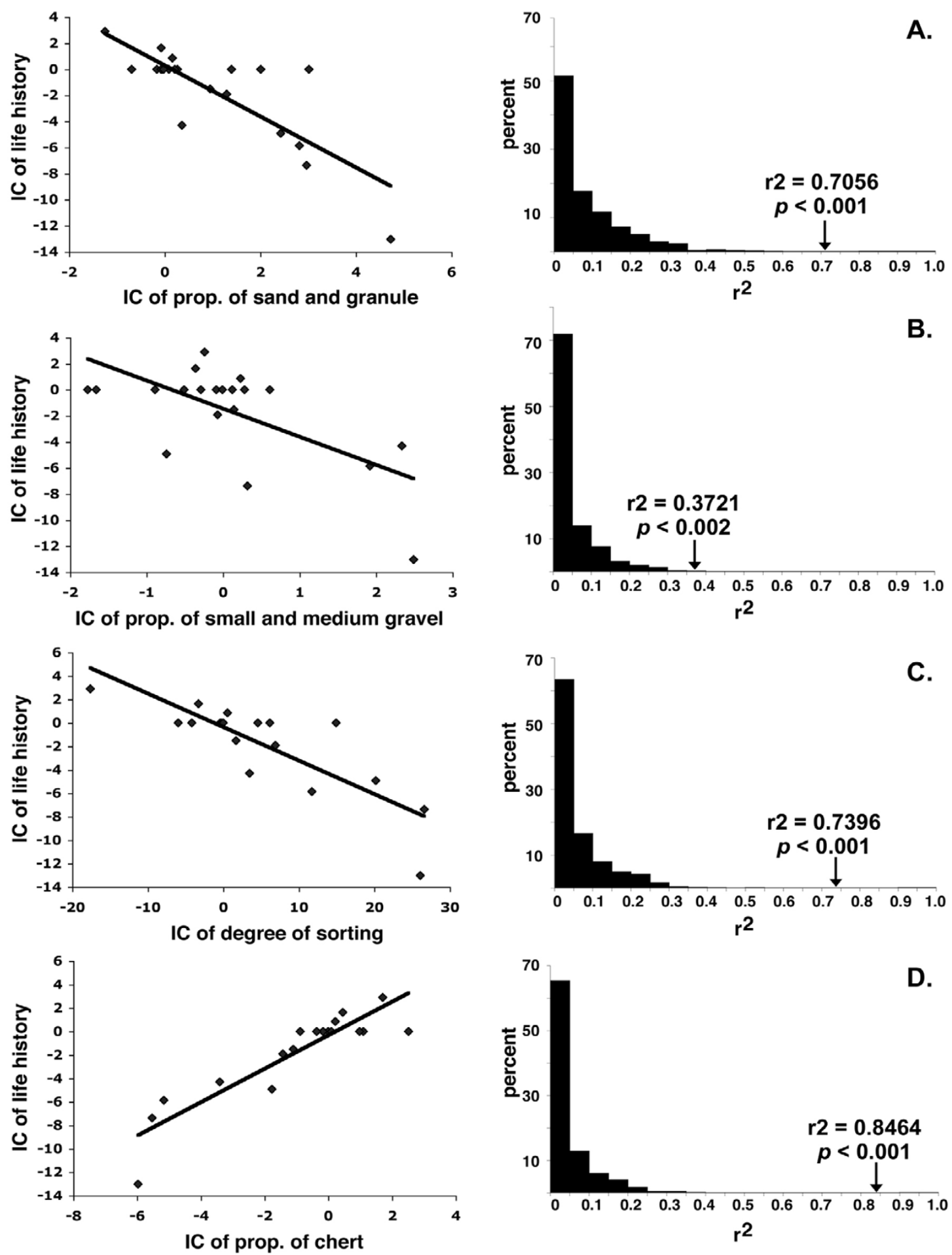

Figure 3

Relationships of substrate parameters to life history mode. Regression plots (left) of independent contrasts of substrate parameters (A, proportion of sand and granule; B, proportion of small/medium gravel; C, degree of sorting; $\mathbf{D}$, proportion of chert) versus independent contrasts of life history mode. Histograms (right) represent the distribution of $r^{2}$ values based on 1000 MCMC randomizations of the original data. Arrows indicate location of observed $r^{2}$ relative to the randomized distribution. 
with chert gravel, but transforming populations, which were thought to be a different species, were not examined in earlier studies [33-35]. Therefore, to quantify substrate type for both life history modes we measured the proportion of the volume of each sample that was composed of chert versus clastic material at each locality. The proportion of chert present in samples ranged from 0 to $97 \%$. Paedomorph localities were primarily composed of chert, while the M localities primarily contained clastic material. We found a strong positive correlation between the presence of chert and paedomorphosis $\left(\mathrm{r}^{2}=0.8464 ; p<0.001\right.$; Fig. 3D). Conversely, metamorphosis was strongly correlated with clastic substrates.

\section{Discussion}

Streambed microstructure and life history of E. tynerensis Previous work has shown the presence of E. tynerensis to be associated with Silurian and Ordovician chert gravel streams in the western Ozark Plateau [33-35]. However, because the authors of these studies considered only paedomorphs as E. tynerensis, they were studying not the distribution of the species but of a life history mode that we now know to be independently derived in different parts of a complex clade. In the light of new evidence that E. tynerensis comprises both paedomorphic and transforming populations [37], we found that this developmental and life history polymorphism is strongly correlated with several substrate parameters (size, degree of sorting and type). These variables create distinctly different streambed microstructures where E. tynerensis occurs (Fig. 4), which has major implications for the evolution of alternate life history modes.

Consistent with previous studies [33-35], we found that paedomorphic populations are primarily restricted to streams with chert gravel, which is very well sorted into large size classes. Various factors have been hypothesized to explain why chert gravel is important for paedomorphic E. tynerensis [33-35]. Chert is an extremely hard substance that breaks into sharp conchoidal patterns, producing highly irregular shapes. When chert gravel is deposited it creates very porous streambeds with large interstitial spaces that can be utilized by small aquatic organisms [33-35]. Previous studies have shown highly embedded streams (containing a high percentage of fine sediment) to be devoid of paedomorphic E. tynerensis $[33,34]$. Owing to the very hard nature of chert, fracturing is the primary mode of decomposition, and very little fine material is released during a break. Furthermore, the irregular shapes and sharp edges of chert have been proposed to promote turbulent currents between interstitial spaces, which further inhibit deposition of fine sediments [35]. In most localities the interstitial spaces between the chert gravel are crucial to the existence of paedomorphic $E$. tynerensis for avoidance of both predators and desiccation [33-
35]. During xeric summer conditions, most small streams across the Ozark Plateau dry completely at the surface. Interstitial spaces provide pathways for small fishes, crayfish, aquatic salamanders and other aquatic organisms to access the water table far below the surface $[29,34,35]$. During extreme drought years, paedomorphic individuals of E. tynerensis have been found at depths as great as 2.5 meters below the surface [29].

We found the substrate parameters for metamorphic populations to be different from those for paedomorphic populations. In general, substrates in localities where $E$. tynerensis metamorphoses contain very little chert gravel and are primarily composed of poorly sorted and relatively smaller clastic (sandstone and siltstone) materials. Clastic rocks are formed from compression of particulate matter such as sand (sandstone) and silt (siltstone). Erosion of clastic rocks causes them to break down gradually into constituents. For sandstone and siltstone, this causes the deposition of a wide range of particle sizes (i.e., poorly sorted material [41]) in streams where these rocks occur. Small sediments fill in the interstitial spaces between larger particles, forming a densely packed streambed microstructure that is largely impenetrable [33]. Therefore the substrate blocks access to any potential subterranean water, so when surface water dries, metamorphosis is presumably critical at these localities. Metamorphic E. tynerensis have a relatively brief larval period (seven to eight months) that matches the seasonality of surface habitat moisture in the Ozark Plateau [42].

Owing to its direct connection with subsurface water, the microstructure of streambed sediments appears to be a major habitat feature determining life history mode in $E$. tynerensis, although it may not be the only factor. Nutrient abundance, persistence of underground water, availability of suitable peripheral habitat, or many other factors may also influence the distribution of alternate life history modes in this group. The terrestrial habitats surrounding paedomorphic E. tynerensis localities are often very dry, because porous chert streambeds that extend outside the stream proper are unable to sustain moisture. The lack of suitable peripheral habitat for metamorphosing salamanders probably favours selection for paedomorphosis, or the ability to shift facultatively to this life-history mode. A similar situation occurs in the hot, dry Edwards Plateau of central Texas: naturally metamorphosing populations of Eurycea (members of the E. troglodytes complex sensu $[43,44])$ appear limited to a small region of mesic canyons with moist streamside habitat, whereas all other known Eurycea in the region are strictly paedomorphic $[44,45]$.

The relationships among all the 26 recognized species of Eurycea [46] are unclear, but at least one highly relevant 


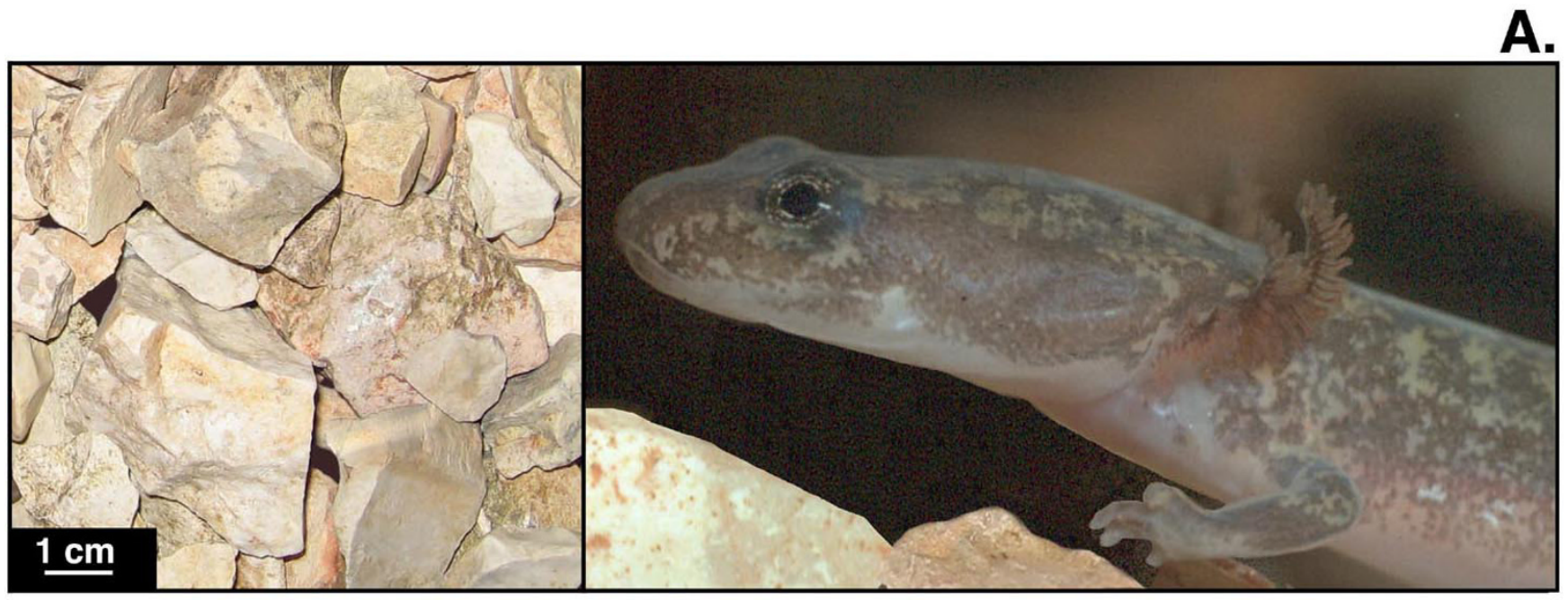

B.

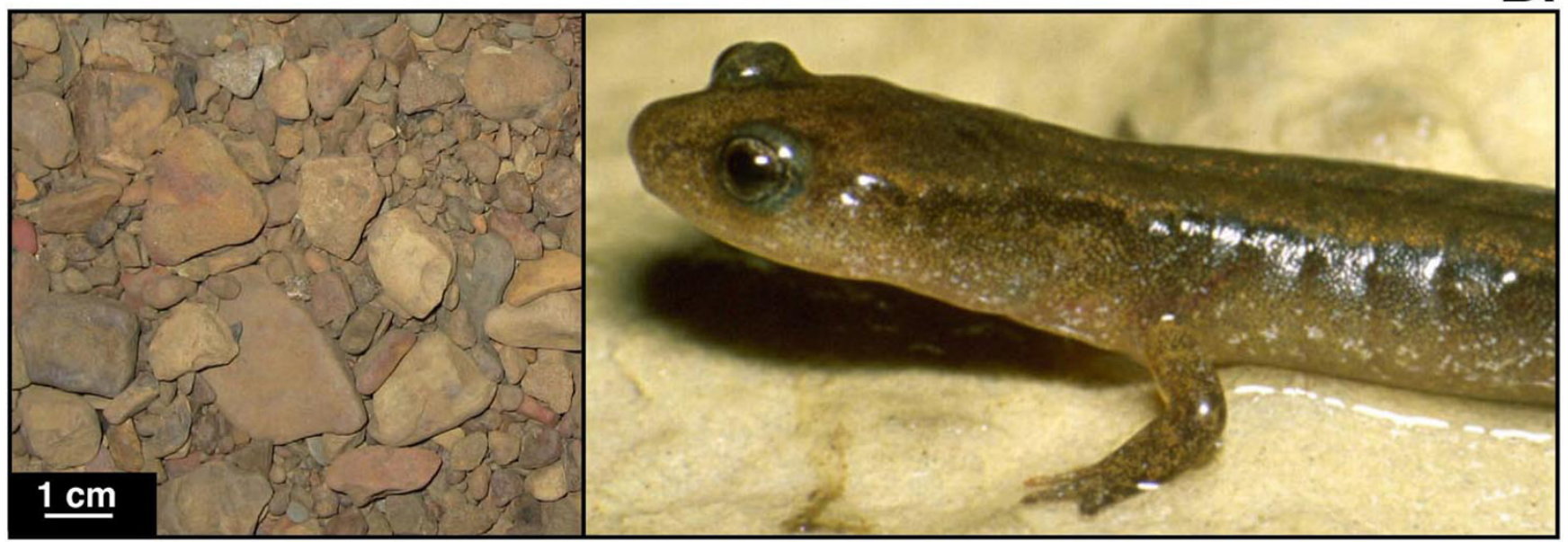

\section{Figure 4}

Alternate life history modes of $\mathbf{E}$. tynerensis and associated substrates. A, Paedomorphic Eurycea tynerensis and an example of Silurian/Ordovician chert gravel from sites where paedomorphs occur. Note the large particle sizes that provide interstitial spaces in the substrate. B. Metamorphosed $E$. tynerensis and an example of densely packed and poorly sorted clastic rock typical of metamorphic habitat. Scale bars only apply to the substrate pictures. Salamander photographs compliments of R. W. Van Devender.

aspect of the phylogeny has been established. Eurycea tynerensis is phylogenetically nested in a clade of otherwise strictly metamorphosing species (E. multiplicata and E. spelaea) $[37,47]$. Thus, paedomorphosis, or the ability to be facultatively paedomorphic, probably evolved independently in the ancestor of the E. tynerensis group, while the ability to metamorphose has also been maintained. Paedomorphosis enables the exploitation of unique chert gravel bottom streams that allow continuous access to permanent water, while metamorphosis has permitted the continued colonization of seasonally ephemeral aquatic habitats throughout the Ozark Plateau. Several other species of Eurycea (E. longicauda, E. lucifuga, and E. spelaea) are broadly sympatric with E. tynerensis, however none exhibit paedomorphosis [48]. This may explain their restriction to the headwaters of springs and to caves that contain water, whereas the distribution of E. tynerensis extends much further throughout the stream [49]. This further supports the hypothesis that some independently evolved feature of the E. tynerensis clade governs paedomorphosis.

Here we treat E. tynerensis as a single species, the most conservative approach given the well-supported monophyly of the group. However, especially given the levels of sequence divergence present, it seems likely that numerous independent lineages are evolving in response to 
readily quantifiable habitat parameters, and multiple species may already be present. Whether because phenotypic plasticity is retained over long periods of evolutionary time or metamorphosis versus paedomorphosis is repeatedly selected for, members of the E. tynerensis group represent a clear example of evolutionary developmental change in vertebrates attributable to differences in habitat microstructure.

\section{Developmental divergence in salamanders}

Nine of the 10 salamander families exhibit paedomorphosis $[10,14,48]$, a developmental deviation from the typically biphasic amphibian life cycle to a strictly aquatic one. All species in the families Amphiumidae, Cryptobranchidae, Proteidae, and Sirenidae are paedomorphic, whereas within five other families (Ambystomatidae, Dicamptodontidae, Hynobiidae, Plethodontidae, and Salamandridae), paedomorphosis is variable within and among species. All species of amphiumids, cryptobranchids, proteids, and sirenids, as well as some plethodontids and ambystomatids, are obligate paedomorphs (i.e., cannot metamorphose) in nature $[10,12,14]$. For many of these species the ability to metamorphose has been completely lost, and cannot even be induced experimentally with hormonal treatment $[12,14]$. Conversely, several species of ambystomatids, salamandrids, and possibly other salamanders are facultatively paedomorphic, with life-history mode dictated by environmental conditions [10]. This plasticity allows populations, and even individuals, to change strategies when faced with fluctuating environmental conditions. Individuals of some species may be paedomorphic for part of their life (i.e. reproduce in the aquatic larval form) prior to metamorphosis $[48,50]$.

Selection for plasticity in life-history strategies allows individuals, populations and/or species to persist in unpredictable environments [7]. However, selection on populations in temporally very stable environments may favour a single mode of life history and eliminate another $[21,23,51]$. This seems to be the case for several paedomorphic populations of ambystomatids $[20,26,28,52]$, salamandrids $[17,24]$, and plethodontids $[[27,53]$, this study] that inhabit relatively permanent aquatic systems, where surrounding terrestrial conditions often are harsh and not conducive for terrestrial salamanders. Arguably, temporary aquatic habitats strongly select for metamorphosis in salamanders with an aquatic larval stage [[50,51], this study]. Genetic crosses [54,55] and molecular genetic analyses [56-59] indicate that there are strong heritable components to the variations in developmental timing of closely related ambystomatid salamanders. Artificial selection studies on Ambystoma talpoideum demonstrated that pond drying can significantly increase the frequency of metamorphosis over very brief periods (only four generations) [51]. Toward the opposite extreme, obligately paedomorphic salamanders may arise from facultatively paedomorphic ancestors $[15,20]$. For example, based on genetic linkage mapping in captive versus wild populations of the axolotl (Ambystoma mexicanum), Voss and Shaffer [57] suggested that obligate paedomorphosis in captive populations may have resulted from artificial selection and/or bottlenecking when the colonies were established. The seemingly irreversible evolution of obligate paedomorphosis may be the result of either strong selection for paedomorphic "alleles", or loss-of-function mutations in genes associated with metamorphosis. Consequently, the removal of ecological, developmental or genetic constraints associated with metamorphosis or terrestriality may have allowed for the evolution of some of the most morphologically divergent salamanders. Aquifer-dwelling paedomorphic plethodontids such as Eurycea rathbuni and Haideotriton wallacei have extreme subterranean morphologies, and bear little resemblance to relatively closely related surface-dwelling species $[43,44,48,60]$. Indeed, the obligate paedomorphic salamanders of the families Sirenidae and Amphiumidae, each of which diverged from other salamanders by at least the Upper Cretaceous [61], have evolved to be similar in body form and ecology to "eel like" fishes and dipnoans.

\section{Life history evolution and reproductive isolation in plethodontids}

More than two thirds (377 described species) of the world's salamanders are in the Family Plethodontidae [46], a group marked by extensive variation in ecology, life history, developmental timing and morphological innovation [53,62-64]. This diversity has been attributed, in part, to relatively low dispersal rates among populations [65], combined with divergence in courtship behaviours and evolution of associated pheromones [66-70]. These important aspects of plethodontid biology lead us to propose that reproductive isolation among populations exhibiting alternate developmental modes may occur more readily in plethodontids than other salamanders. The Eurycea tynerensis group will be a useful system for testing this hypothesis.

Morphological development and courtship behaviour are decoupled in the salamandrid Triturus alpestris [71,72], and paedomorphic and metamorphic individuals can court and reproduce despite having divergent morphologies. However, we suggest that the developmental shift from metamorphosis to paedomorphosis in plethodontids could have a profound impact on mate recognition. Almost all metamorphosing plethodontids exhibit terrestrial courtship, a strategy that is uncommon among the rest of the salamander families [66-70,73]. One diagnostic feature of adult metamorphosing plethodontids that is intimately associated with terrestrial courtship is the 
nasolabial groove, a tract on the surface of the skin that leads from the nostril to the margin of the upper lip [6670,73 . To the best of our knowledge, the nasolabial groove only develops completely in fully metamorphosed individuals [[74-78], RMB pers obs, D. B. Wake pers com]. Therefore, we speculate that paedomorphic individuals may retain the larval aquatic communication system, while terrestrial metamorphs use other methods to find mates.

Spatial segregation in breeding ponds creates asymmetric reproductive isolation between sympatric morphs of the mole salamander, Ambystoma talpoideum [79]. Metamorphic males rarely encounter paedomorphic females, but no isolation is apparent between paedomorphic males and metamorphic females [79]. This asymmetry is considered to have little influence on sympatric speciation of the alternate phenotypes [79]. Little is known about the reproductive biology of Eurycea tynerensis, but there are notable differences between paedomorphic and metamorphosing populations $[42,80]$. For example, in many localities courting pairs of transformed individuals are commonly found at the edges of streams out of water, where it would be impossible for paedomorphs to reproduce [RMB pers obs]. Consequently, in addition to possible selection for single, distinct life history modes among populations, ecological, structural and behavioral differences could present barriers to communication between paedomorphs and metamorphs, promoting reproductive isolation in any areas where these two forms may meet. This could provide the basis for speciation.

\section{Conclusion}

The geologically diverse Ozark Plateau is a unique landscape where distinctly different stream habitats occur in very close proximity. These habitat differences appear to strongly influence the development of $E$. tynerensis, either facultatively or by selection for alleles governing alternate developmental strategies, as most populations are unimodal in life history. The remarkably robust association between a relatively simple habitat feature such as streambed microstructure and developmental mode in salamanders of the Eurycea tynerensis group represents strong evidence for the role of microhabitat factors that may ultimately determine major changes in ontogeny, ecology, morphology and life history, and contribute to the process of speciation.

\section{Methods}

\section{Sampling localities and specimens}

Eurycea tynerensis were collected from throughout the Ozark Plateau of Arkansas, Missouri and Oklahoma by RMB and colleagues from August 2000 - August 2003. We selected 22 localities (11 paedomorphic and 11 metamorphic; Fig. 1; [see Additional File 1]) from throughout the geographic distribution, which represent the full spectrum of mitochondrial divergence within E. tynerensis [37]. Adult specimens from each of the 22 localities were collected for phylogenetic analyses. Salamanders were euthanised by submersion in a $10 \%$ solution of MS-222 according to IACUC protocols. Vouchers and tissues are in the University of Texas at Arlington (UTA) Amphibian and Reptile Diversity Research Center. Over a five year period (2000-2005) each of these localities was visited multiple times, and adult individuals observed at each locality were either all paedomorphic (sexually mature in the aquatic larval form) or all metamorphic (completely transformed). Sexual maturity of paedomorphs was determined by the presence of oviducal eggs in females and well-developed pigmented testes in males. At all the metamorphic localities, females guarding eggs and/or immature larvae were found on at least one visit, indicating that metamorphic E. tynerensis were breeding along these streams and were not simply transients.

\section{DNA isolation amplification and sequencing}

DNA was isolated using DNeasy extraction kits (Qiagen). Portions of two mitochondrial genes, cytochrome $b$ ( $c o b$, 1118 nucleotides) and NADH dehydrogenase subunit 4 (nad4, 700 nucleotides), were amplified via polymerase chain reaction (PCR). The primer pairs MVZ15 5'- GAA CTA ATG GCC CAC ACW WTA CGN AA -3' [81] and ETCR 5'- TTC TAA ACT ACA ACA GCA TC -3' [37] were used to amplify $c o b$, and ND4F 5'- CAC CTA TGA CTA CCA AAA GCT CAT GTA GAA GC -3' [82] and EML1R 5'CTT TCR TRT CTA GGG TCA CAG CCT AG -3' [37], were used for nad4. PCR products were electrophoresed on $1 \%$ agarose gels. Bands of the expected molecular weight were excised, purified and ligated into a plasmid vector using Topo-TA kits (Invitrogen). Plasmids were used to transform $E$. coli via heat shocking at $42^{\circ} \mathrm{C}$. Transformed cells were plated on kanamycin/X-gal plates for blue/white selection. White colonies were picked and cultured overnight in Luria-Bertani broth. Plasmids were purified using a Qiagen Plasmid Miniprep kit and standard protocols, except that a 1:10 dilution of EB buffer:/water was used for the final elution. Approximately $4 \mu \mathrm{l}$ of plasmid was dried for sequencing. SequiTherm Excel ${ }^{\mathrm{TM}}$ sequencing kits (Epicentre Technologies) and infrared labeled primers were used for sequencing reactions. Plasmids from at least two different clones of each fragment, for each specimen, were bi-directionally sequenced on a LiCor 4200 L long-read, dual-laser sequencer. Both strands were sequenced completely for each sample.

\section{Sequence alignment and phylogenetic analyses}

We used Sequencher ${ }^{\mathrm{TM}} 3.1$ (Gene Codes Corp.) to align and edit sequences. No indels were observed, and alignment was unambiguous. Both gene fragments were translated in MacClade [83], and no stop codons were found. 
Sequences were deposited in GenBank [see Additional File 1]. A Nexus file containing a concatenated alignment of both genes was analyzed using Bayesian methodology. Eurycea multiplicata and E. spelaea, two species closely related to E. tynerensis $[37,47]$, were used as outgroups to root the trees. ModelTest v. 3.06 [84] was used to calculate the most appropriate model of nucleotide substitution. ModelTest selected the same model $(\mathrm{HKY}+\mathrm{I}+\Gamma)$ for both genes. For $c o b$, transition/transversion $(\mathrm{Ti} / \mathrm{Tv})$ ratio $=$ 8.4750, proportion of invariable sites $(\mathrm{I})=0.4856$, gamma distribution shape parameter $(\Gamma)=0.8822$, and mean base frequencies were $\mathrm{A}=0.3078 ; \mathrm{C}=0.2557 ; \mathrm{G}=$ $0.1341 ; \mathrm{T}=0.3024$. For $n a d 4, \mathrm{Ti} / \mathrm{Tv}$ ratio $=11.2622, \mathrm{I}=$ $0.3496, \Gamma=0.3835$, and mean base frequencies were $\mathrm{A}=$ $0.3404 ; \mathrm{C}=0.2768 ; \mathrm{G}=0.1179 ; \mathrm{T}=0.2649$.

The data were partitioned by gene ( $c o b$ and nad4) for Bayesian analyses (all partitions unlinked) implemented in MrBayes 3.0 [85]. Analyses were run with four chains and uniform priors for 5.05 million generations (with the first 50,000 discarded as burn-in; stationarity of likelihoods achieved before this point). The resulting 50\% majorityrule consensus of the 50,000 post burn-in trees, sampled every 100 generations, was computed in PAUP*v $4.0 \mathrm{~b} 10$ [86]. Bayesian branch lengths for the combined data set were averaged across trees in MrBayes 3.0 [85]. Unweighted maximum parsimony analysis and nonparametric bootstrapping were also performed using PAUP* . The resulting tree was congruent in most respects with the Bayesian tree, with strong support for the same nodes that were highly supported in the Bayesian analysis. Phylogenetic independent contrasts (see below) using topologies and branch lengths from both methods of analyses yielded nearly identical results. Given the high degree of congruence between the results of both analyses, we only show the results from Bayesian analyses.

\section{Substrate sampling and quantification of size and type}

Three stream substrate samples were collected from each of the 22 localities. The first substrate sample was collected immediately adjacent to where the first E. tynerensis at each locality was found during the sampling period. The other two samples were randomly selected from one to six meters up and downstream from where the first substrate sample was collected. The number of meters (1 to 6) was decided by rolling a six-sided die. Substrate samples were returned to the University of Texas at Arlington and dried completely under incandescent lamps. Dried substrate samples were sorted by size using a cylinder of eight hierarchically stacked screen sieves. Substrate samples were poured into the top of the cylinder, which was then capped and fixed to an orbit shaker (Lab Line Instruments Inc.) and shaken at $250 \mathrm{rpm}$ for five minutes. The sieves separated the substrate into eight size classes $(0$ to $<0.25$, 0.25 to $<0.5,0.5$ to $<2,2$ to $<4,4$ to $<6,6$ to $<12,12$ to
$<25, \& \geq 25 \mathrm{~mm}$ ) based on mesh diameter. The total volume of substrate (in $\mathrm{ml}$ ) in each category was measured by water displacement in graduated cylinders. The three substrate samples collected at each locality were combined on the basis of their extreme similarity.

\section{Independent contrasts}

Testing for correlations between organismal traits and their environments is one of the many uses of phylogenetic comparative methods [38-40]. We used phylogenetic independent contrasts (PIC) [38] calculated in COMPARE version 4.6 [87] to account for possible phylogenetic non-independence of our data, when testing for a correlation between stream substrate parameters (size, degree of sorting and type) and life history mode. We based our comparative analysis on the Bayesian phylogeny and branch lengths, although results based on the maximum parsimony tree were almost identical (data not shown). Our Bayesian topology had two trichotomies. However, COMPARE cannot handle polytomies [87]. To resolve these ambiguities we arbitrarily placed $\mathrm{P} 8$ as sister to the clade containing $\mathrm{M} 8, \mathrm{P} 9, \mathrm{M} 7, \mathrm{P} 7, \mathrm{P} 10$ and $\mathrm{P} 11$, and we made P5 and P6 a clade. We assigned extremely short ( 0.000001 substitutions/site) lengths to internal branches subtending these nodes [87].

Life history of salamanders at each locality was scored as a categorical variable: paedomorphic (0) and metamorphic (1). PIC of life history mode was calculated against four continuous substrate parameters (the proportion of sand and granule, the proportion of small/medium gravel, the number of substrate size classes, and the proportion of chert) for the 22 localities. Standard errors of all variables were set to zero. Using SAS v. 8.2 (SAS institute Inc.), 1000 Markov Chain Monte Carlo (MCMC) random iterations were used to create a random distribution of $\mathrm{r}^{2}$ values based on the original data. To assess significance $(\mathrm{p}<0.05)$, our observed $\mathrm{r}^{2}$ for each regression was compared to this distribution to test the relationship between life-history mode and a given substrate parameter. We also found streams containing large gravel to be positively correlated with paedomorphosis, but we do not formally test this because it is simply an "inverse" test of sand and granule association with developmental mode.

The original application of phylogenetic comparative methods such as independent contrasts was for interspecific comparisons, although intraspecific studies have highlighted the utility of these methods within species [88-90]. Recently, Niewiaroski and others [91] questioned the necessity of applying phylogenetic comparative methods below the species level. They pointed out the possibility of making Type I errors (overlooking significant correlations) by using such a conservative method at shallow levels of divergence, but noted that more studies at 
this scale are warranted. Even though phylogenetic comparative methods may be statistically conservative, we implement them here to be certain that our results are not biased by common ancestry.

\section{Abbreviations}

cob, mitochondrial gene cytochrome $\mathrm{b} ; \mathrm{M}$, metamorphic; nad4, mitochondrial gene NADH dehydrogenase subunit 4; P, paedomorphic; PICs, phylogenetic independent contrasts; UTA, University of Texas at Arlington.

\section{Authors' contributions}

$\mathrm{RMB}$ conceived the experiment, collected and analyzed the data, and primarily prepared the manuscript. The work was conducted in the laboratory of PTC, who contributed to the study design, analyses and manuscript preparation.

\section{Additional material}

\section{Additional File 1}

Locality information, and museum and Genbank accession numbers for the 22 populations of $\mathrm{E}$. tynerensis and outgroups.

Click here for file

[http://www.biomedcentral.com/content/supplementary/1741-

7007-4-6-S1.pdf]

\section{Acknowledgements}

We thank D. Fenolio, M. Gerson and J. Wiens for joining us in the field. J. Briggler, W. Elliott and K. Irwin facilitated many phases of this study. A. Baskin, N. Bendik, K. Ivanova, B. Noonan and M. Trussell were of great assistance in the lab. D. Reaser and J. Mergele (Department of Geology, UTA) verified substrate identification. D. Buckley, M. Gerson, W. Korff, J. MacKenzie, J. Marshall, E. Martins, I Martinéz, P. Michalak, B. Noonan, R. Tumlison, D. Vieites, M. Wake, D. Wake and two anonymous reviewers provided valuable insight on various aspects of this study. The Arkansas Game and Fish Commission, Missouri Department of Conservation and Oklahoma Department of Wildlife Conservation permitted specimen collection. This project was supported by NSF Doctoral Dissertation Improvement Grant DEB-0206730 to RMB and PTC, a Gaige Fund Award to RMB from the American Society of Ichthyologists and Herpetologists, additional NSF funding (DEB-0 29242) to PTC, and NSF post-doctoral support (EF-0334939) for RMB.

\section{References}

I. Stearns SC: The evolution of life histories New York, USA: Oxford University Press; 1992.

2. Schluter D: The ecology of adaptive radiation New York, USA: Oxford University Press; 2000.

3. Schluter D: Ecology and the origin of species. Trends Ecol Evol 200I, 16:372-380.

4. Dewitt TJ, Scheiner SM: Phenotypic plasticity: functional and conceptual approaches New York, USA: Oxford University Press; 2004.

5. Rollo CD: Phenotypes: their epigenetics, ecology and evolution London, UK: Chapman and Hall; 1994.

6. Jablonka $E$, Szathmary $E$ : The evolution of information storage and heredity. Trends Ecol Evol 1995, 10:206-2II.

7. Gould SJ: Ontogeny and phylogeny Cambridge (Massachusetts, USA): Harvard University Press; 1977.
8. West-Eberhard MJ: Developmental plasticity and evolution New York, USA: Oxford University Press; 2003.

9. Moczek AP: Developmental plasticity and the origins of diversity: a case study on horned beetles. In Insects and phenotypic plasticity Edited by: Ananthakrishnan TN, Whitman D. United Kingdom: Science Publishers Inc; 2003.

10. Duellman WE, Trueb LT: Biology of amphibians Baltimore (Maryland, USA): The John Hopkins University Press; 1986.

II. Wilbur HM, Collins JP: Ecological aspects of amphibian metamorphosis. Science 1973, 182:|305-13|4.

12. Denver RJ, Glennemeier KA, Boorse GC: Endocrinology of complex life cycles: amphibians. In "Hormones, brain and behavior" Volume 2. Edited by: Pfaff D, Arnold A, Etgen A, Fahrbach S, Moss R, Rubin R. San Diego (California, USA): Academic Press Inc; 2002:469-5I3.

13. Bruce RC: Life histories. In Reproductive biology and phylogeny of Urodela Edited by: Sever DM. Enfield (New Hampshire, USA): Science Publishers; 2003:477-525.

14. Dent JN: A survey of amphibian metamorphosis. In Metamorphosis: a problem in developmental biology Edited by: Etkin W, Gilbert LI. (New York, USA): Appleton Century Crofts; I968:27I-3II.

15. Denöel M, Joly P, Whiteman HH: Evolutionary ecology of facultative paedomorphosis in newts and salamanders. Biol Rev 2005, 80:663-671.

16. Ryan TJ, Semlitsch RD: Intraspecific heterochrony and life history evolution: decoupling somatic and sexual development in a facultatively paedomorphic salamander. Proc Natl Acad Sci 1998, 95:5643-5648.

17. Denöel M, Joly P: Neoteny and progenesis as two heterochronic processes involved in paedomorphosis in Triturus alpestris (Amphibia: Caudata). Proc R Soc Lond Ser B 2000, 267:1481-1485.

18. Semlitsch RD: Relationship of pond drying to the reproductive success of the salamander Ambystoma talpoideum. Copeia 1987, 1987:61-69.

19. Semlitsch RD, Wilbur HM: Effects of pond drying time on metamorphosis and survival in the salamander Ambystoma talpoideum. Copeia 1988, 1988:978-983.

20. Schaffer HB: Evolution in a paedomorphic lineage. I. An electrophoretic analysis of Mexican ambystomatid salamanders. Evolution 1984, 38: I 194-I 206.

21. Harris RN: Density-dependent paedomorphosis in the salamander Notophthalmus viridescens dorsalis. Ecology 1987, 68:705-712.

22. Semlitsch RD: Paedomorphosis in Ambystoma talpoideum: effects of density, food, and pond drying. Ecology 1987, 68:994-1002.

23. Semlitsch RD, Harris RN, Wilbur HM: Paedomorphosis in Ambystoma talpoideum: maintenance of population variation and alternate life-history pathways. Evolution 1990, 44:1604-1613.

24. Denoël M, Duguet R, Dzukit G, Kalezic M, Mazzotti S: Biogeography and ecology of paedomorphosis in Triturus alpestris (Amphibia, Caudata). J Biogeography 200I, 29:1217-1280.

25. Krenz JD, Sever DM: Mating and ovoposition in paedomorphic Ambystoma talpoideum proceeds the arrival of terrestrial males. Herpetologica 1995, 5 I:387-393.

26. Sprules WG: The adaptive significance of paedogenesis in North American species of Ambystoma (Amphibia: Caudata): an hypothesis. Canadian Journal of Zoology 1974, 52:393-400.

27. Bruce RC: Population structure, life history, and evolution of paedogenesis in the salamander Eurycea neotenes. Copeia 1976, 1976:242-249.

28. Smith HB: The Mexican axolotl: some misconceptions and problems. BioScience 1969, 19:593-597.

29. Dowling HG: Geographic relations of Ozarkian amphibians and reptiles. The Southwestern Naturalist 1956, I: 174-189.

30. Mayden RL: Vicariance biogeography, parsimony, and evolution in North American freshwater fishes. Syst Biol 1988, 37:329-355.

31. Crandall KA, Tempelton AR: The zoogeography and centers of origin of the crayfish subgenus Procericambarus (Decapoda: Cambaridae). Evolution 1999, 53:123-134.

32. Fenneman NM: Physiography of eastern United States New York, USA: McGraw-Hill Inc; 1938. 
33. Tumlison R, Cline RG, Zwank P: Surface habitat associations of the Oklahoma salamander (Eurycea tynerensis). Herpetologica 1990, 46: 169-I75.

34. Cline RG, Tumlinson R: Distribution and relative abundance of the Oklahoma salamander (Eurycea tynerensis). Proc Okla Acad Sci 1997, 8I:I-I0.

35. Tumlinson R, Cline RG: Association between the Oklahoma salamander (Eurycea tynerensis) and Ordivician-Silurian strata. The Southwestern Naturalist 2003, 48:93-95.

36. Moore GA, Hughes RC: A new plethodontid from eastern Oklahoma. Am Midl Nat 1939, 22:696-699.

37. Bonett RM, Chippindale PT: Speciation, phylogeography and evolution of life history and morphology in plethodontid salamanders of the Eurycea multiplicata complex. Mol Ecol 2004, | 3: | | 89-| 203.

38. Felsenstein J: Phylogenies and the comparative method. Am Nat 1985, I 25:1-15.

39. Garland T Jr, Harvey PH, Ives AR: Procedures for the analysis of comparative data using phylogenetically independent contrasts. Syst Biol 1992, 41:18-32.

40. Martins EP: Adaptation and the comparative method. Trends Ecol Evol 2000, I 5:296-299.

41. Compton RR: Geology in the field New York, USA: John Wiley and Sons; 1985.

42. Ireland PH: Reproduction and development of the gray-bellied salamander, Eurycea multiplicata griseogaster. Herpetologica 1976, 32:233-238.

43. Chippindale PT: Species boundaries and species diversity in the central Texas hemidactyliine plethodontid salamanders, genus Eurycea. In The biology of plethodontid salamanders Edited by: Bruce RC, Jaeger RG, Houck LD. New York, USA: Kluwer Academic, Plenum Publishers; 2000: 149-165.

44. Chippindale PT, Price AH, Wiens JJ, Hillis DM: Phylogenetic relationships and systematic revision of central Texas hemidactyliine plethodontid salamanders. Herpetological Monographs 2000, | 4: 1-80

45. Sweet SS: Natural metamorphosis in Eurycea neotenes and the generic allocation of Texas Eurycea (Amphibia; Plethodontidae). Herpetologica 1977, 33:364-375.

46. AmphibiaWeb: Information on amphibian biology and conservation [http://amphibiaweb.org/]. Berkeley, California

47. Bonett RM, Macey JR, Boore JL, Chippindale PT: Resolving the tips of the Tree of Life: How much mitochondrial data do we need? A case study in plethodontid salamanders. . in review

48. Petranka JW: Salamanders of the United States and Canada Washington DC, USA: Smithsonian Institution Press; 1998.

49. Rudolph DC: Aspects of the larval ecology of five plethodontids of the western Ozarks. Am Midl Nat 1978, 100:141-159.

50. Semlitsch RD, Gibbons JW: Phenotypic variation in metamorphosis and paedomorphosis in the salamander Ambystoma talpoideum. Ecology 1985, 66: II23-1।30.

51. Semlitsch RD, Wilbur HM: Artificial selection for paedomorphosis in the salamander Ambystoma talpoideum. Evolution 1989, 43:105-112.

52. Snyder RC: Comparative features of the life histories of Ambystoma gracile (Baird) from populations at low and high altitudes. Copeia 1956, 1956:41-50.

53. Ryan TJ, Bruce RC: Life history evolution and adaptive radiation of hemidactyliine salamanders. In The biology of plethodontid salamanders Edited by: Bruce RC, Jaeger RG, Houck LD. New York, USA: Kluwer Academic, Plenum Publishers; 2000:303-325.

54. Harris RN, Semlitsch RD, Wilbur HM, Fauth JE: Local variation in the genetic basis of paedomorphosis the salamander Ambystoma talpoideum. Evolution 1990, 44:I588-I603.

55. Voss RA: Genetic basis of paedomorphosis in the axolotl, Ambystoma mexicanum: a test for the single gene hypothesis. I Heredity 1995, 86:441-447.

56. Voss RA, Shaffer HB: Adaptive evolution via a major gene effect: paedomorphosis in the Mexican axolotl. Proc Natl Acad Sci USA 1997, 94:14185-14189.

57. Voss RA, Shaffer HB: Evolutionary genetics of metamorphic failure using wild-caught vs. laboratory axolotl (Ambystoma mexicanum). Mol Ecol 2000, 9: |40 I- 407.

58. Voss RA, Prudic KL, Oliver JC, Shaffer HB: Candidate gene analysis of metamorphic timing in ambystomatid salamanders. Mol Ecol 2003, 12:1217-1223.
59. Voss RA, Smith J]: Evolution of salamander life cycles: A major effect QTL contributes to discrete continuous variation for metamorphic timing. Genetics 2005, I 70:275-28I.

60. Wiens J], Chippindale PT, Hillis DM: When are phylogenetic analyses misled by convergence? A case study in Texas cave salamanders. Syst Biol 2003, 52:50I-5I4.

61. Milner AR: Mesozoic and Tertiary Caudate and Albanerpetontidae. In Amphibian biology, Paleontology and evolutionary history of amphibians Volume 4. Edited by: Heatwole H, Carroll RL. NSW: Chipping Norton; 2000: I4I2-I444.

62. Wake DB: Comparative osteology and evolution of the lungless salamanders, Family Plethodontidae. Mem So Cal Acad Sci 1966, 4: I- III

63. Wake DB, Hanken J: Direct development in the lungless salamanders: what are the consequences for developmental biology, evolution and phylogenesis? Int J Dev Biol 1996, 40:859-869

64. Hanken J: Life history and morphological evolution. J Evol Biol 1992, 5:549-557.

65. Larson A, Wake DB, Yanev K: Measuring gene flow among populations having high levels of genetic fragmentation. Genetics 1984, 106:293-308.

66. Arnold SJ: The evolution of courtship behavior in New World salamanders with some comments on Old World salamandrids. In The reproductive biology of amphibians Edited by: Taylor $\mathrm{H}$, Guttman SI. New York, USA: Plenum Publishers; 1977:141-183.

67. Verrell PA, Arnold SJ: Behavioral observations of sexual isolation among allopatric populations of the mountain dusky salamander, Desmognathus ochrophaeus. Evolution 1989, 43:745-755

68. Verrell PA, Mabry M: The courtship of plethodontid salamanders: form, function, and phylogeny. In The biology of plethodontid salamanders Edited by: Bruce RC, Jaeger RG, Houck LD. New York, USA: Kluwer Academic, Plenum Publishers; 2000:37I-380.

69. Houck LD, Arnold SJ: Courtship and mating behavior. In Reproductive biology and phylogeny of Urodela Edited by: Sever DM. Enfield (New Hampshire, USA): Science Publishers; 2003:383-424.

70. Kozak KH: Sexual isolation and courtship behavior in salamanders of the Eurycea bislineata species complex, with comments on the evolution of mental gland and pheromone delivery behavior in the Plethodontidae. Southeastern Naturalist 2003, 2:28I-292.

7I. Denöel M, Poncin P, Ruwet J: Sexual compatability between two heterochronic morphs in the alpine newt, Triturus alpestris. Anim Behav 2001, 62:559-566.

72. Denöel M: Paedomorphosis in the alpine newt (Triturus alpestris): decoupling behavioral and morphological change. Behav Ecol Sociobiol 2002, 52:394-399.

73. Reagan NL, Verrell PA: The evolution of plethodontid salamanders: did terrestrial mating facilitate lunglessness? Am Nat 1991, 138:1307-1313.

74. Wilder IW: The morphology of amphibian metamorphosis Northampton (Massachusetts, USA): Smith College; 1925.

75. Kezer J: Thyroxine-induced metamorphosis of the neotenic salamanders Eurycea tynerensis and Eurycea neotenes. Copeia 1952, 1952:234-237.

76. Dundee HA: Partial metamorphosis induced in Typhlomolge rathbuni. Copeia 1957, 1957:52-53.

77. Dundee HA: Response of the neotenic salamander Haideotriton wallacei to a metamorphic agent. Science 1962 , I35:1060-106|.

78. Rose CS: Skeletal morphogenesis in the urodele skull: postembryonoc development in the Hemidactyliini (Amphibia: Plethodontidae). I Morph 1995, 223:125-148.

79. Whiteman $\mathrm{HH}$, Semlitsch RD: Asymmetric reproductive isolation among polymorphic salamanders. Biol J Linn Soc 2005 , 86:265-28I.

80. Bonett RM: The Oklahoma salamander, Eurycea tynerensis. In Amphibian declines, the conservation and status of United States species Edited by: Lannoo M. Berkeley (California, USA): University of California Press; 2005:767-769.

81. Moritz C, Schneider C], Wake DB: Evolutionary relationships within the Ensatina eschscholtzii complex confirm the ring species interpretation. Syst Biol 1992, 41:273-291.

82. Arévalo E, Davis SK, Sites JW: Mitochondrial DNA sequence divergence and phylogenetic relationships among eight 
chromosome races of Sceloporus grammicus complex (Phrynosomatidae) in central Mexico. Syst Biol 1994, 43:387-4I8.

83. Maddison DR, Maddison WP: MacClade. Analysis of phylogeny and character evolution, v. 4.03 Sunderland (Massachusetts, USA): Sinauer Associates; 200I.

84. Posada D, Crandall KA: Modeltest: testing the model of DNA substitution. Bioinformatics 1998, 14:817-818.

85. Huelsenbeck JP, Ronquist FR: MrBayes 3.0 Bayesian inference of phylogeny. Bioinformatics 200I, I7:754-755.

86. Swofford DL: PAUP*: Phylogenetic Analysis Using Parsimony and Other Methods. v. 4.0b/0 Sunderland (Massachusetts, USA): Sinauer Associates; $200 \mathrm{I}$.

87. Martins EP: COMPARE: Computer programs for the statistical analysis of comparative data. v. 4.62004 [http://compare.bio.indiana.edu/]. Bloomington (Indiana, USA): Department of Biology, Indiana University

88. Edwards SV, Kot M: Comparative methods at the species level: geographic variation in morphology and group size in greycrowned babblers (Pomatostomus temporalis). Evolution 1995, 49: I I34-II 46

89. Zamudio KR: The evolution of female-biased sexual size dimorphism: a population-level comparative study in horned lizards (Phrynosoma). Evolution 1998, 52:182I- 833.

90. Wiens J], Reeder TW, Nieto Montes de Oca A: Molecular phylogenetics and evolution of sexual dichromatism among populations of the Yarrow's spiny lizard (Sceloporus jarrovii). Evolution 1999, 53:1884-1897.

91. Niewiarowski PH, Angilletta MJ, Leache AD: Phylogenetic comparative analysis of life-history variation among populations of the lizard Sceloporus undulatus: an example and prognosis. Evolution 2004, 58:619-633.

Publish with Biomed Central and every scientist can read your work free of charge

"BioMed Central will be the most significant development for disseminating the results of biomedical research in our lifetime. "

Sir Paul Nurse, Cancer Research UK

Your research papers will be:

- available free of charge to the entire biomedical community

- peer reviewed and published immediately upon acceptance

- cited in PubMed and archived on PubMed Central

- yours - you keep the copyright
BioMedcentral 